Supporting Information

\title{
Spherical Neutralizing Aptamer Inhibits SARS-CoV-2 Infection and Suppresses Mutational Escape
}

Miao Sun ${ }^{1+}$, Siwen Liu ${ }^{3+}$, Ting Song ${ }^{1}$, Fude Chen ${ }^{1}$, Jialu Zhang ${ }^{1,2}$, Jia-ao Huang ${ }^{1}$, Shuang Wan ${ }^{1}$, Yao Lu ${ }^{1}$, Honglin $\mathrm{Chen}^{3^{*}}$, Weihong Tan ${ }^{2 *}$, Yanling Song ${ }^{1 *}$, Chaoyong Yang ${ }^{1,2^{*}}$

1. The MOE Key Laboratory of Spectrochemical Analysis and Instrumentation, State Key Laboratory of Physical Chemistry of Solid Surfaces, Department of Chemical Biology, College of Chemistry and Chemical Engineering, Xiamen University, Xiamen, 361005, China

2. Institute of Molecular Medicine, Renji Hospital, School of Medicine, Shanghai Jiao Tong University, Shanghai, 200127, China

3. State Key Laboratory for Emerging Infectious Diseases, InnoHK Centre for Virology, Vaccinology, and Therapeutics, and Department of Microbiology, Li Ka Shing Faculty of Medicine, The University of Hong Kong, Hong Kong SAR 999077, China 


\section{Table of Contents}

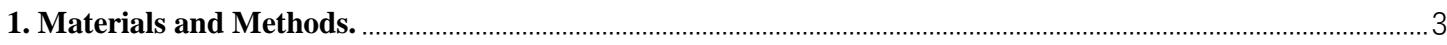

1.1 Reagents.

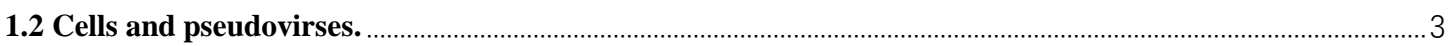

1.3 Construction of AuNPs functionalized with aptamer cocktail (called SNAPs).

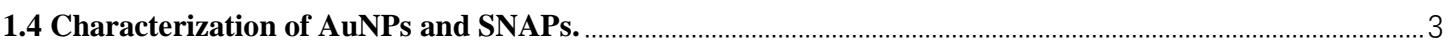

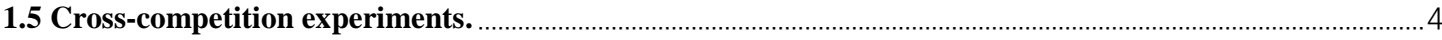

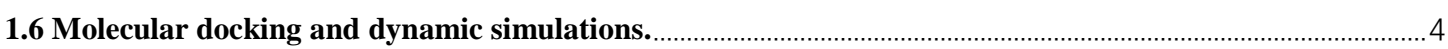

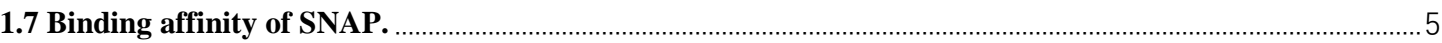

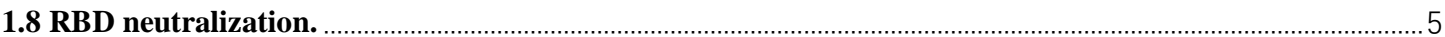

1.9 Stability analysis of aptamer cocktail and SNAP in exonuclease solution. .................................................. 6

1.10 Pseudovirus neutralization analysis.

1.11 Transmission electron microscopy (TEM) and cryo-electron microscopy (cryo-EM) imaging of the

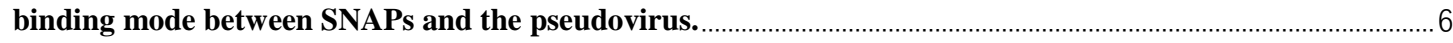

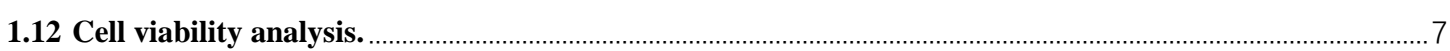

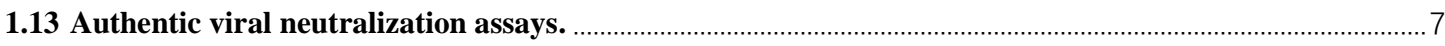

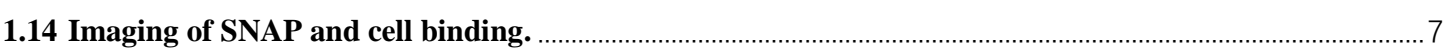

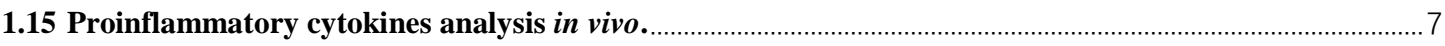

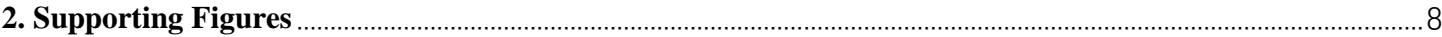

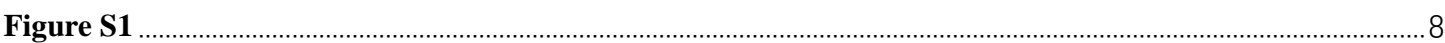

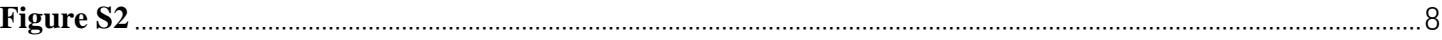

Figure S3

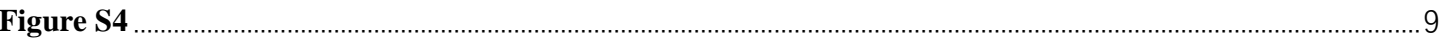

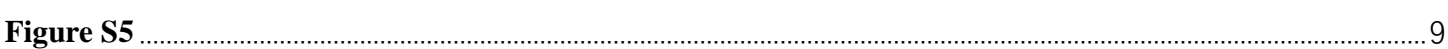

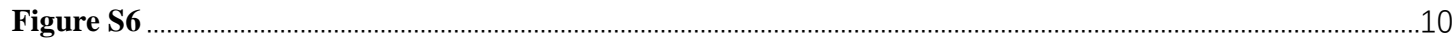

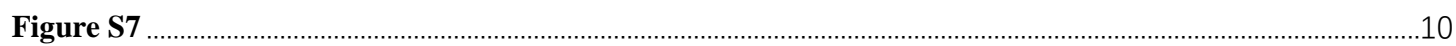

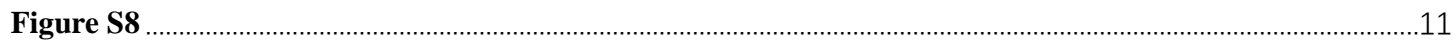

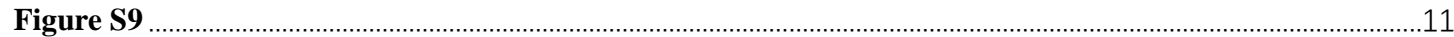

Figure S10

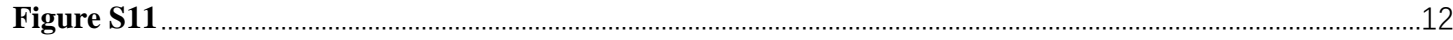

Figure S12

Figure S13

Figure S14

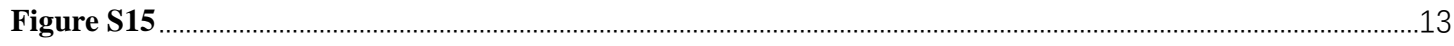

Figure S16

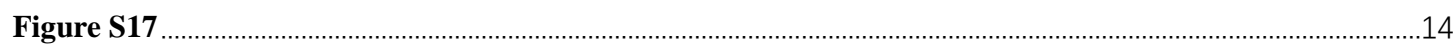

Figure S18

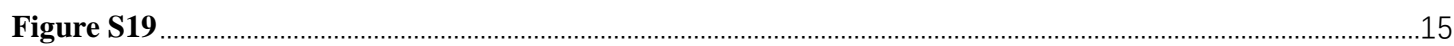

Figure S20

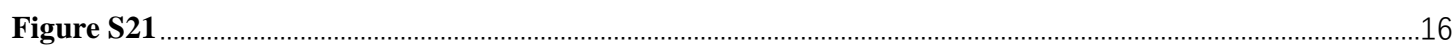


Table S1 Sequences used in this work.

Table S2 Binding Sites of RBD-ACE2, RBD-aptamer, and mutation sites in mutant strains..................................16

Table S3 Variants involved in this work.........................................................................................................17

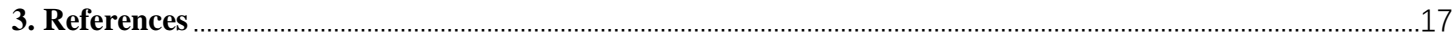

\section{Materials and Methods}

\subsection{Reagents.}

AuNPs (5 nm) were obtained from Sigma-Aldrich (900458). Ni beads for His-tagged RBD or His-tagged ACE2 conjugation were purchased from GE Healthcare (USA). Fc-tagged-RBD of SARS-CoV-2 spike protein (40592V05H), His-tagged-RBD of SARS-CoV-2 spike protein (40592-V08B), SARS-CoV-2 spike S1+S2 ECD-His recombinant protein (40589-V08B1), His-tagged-human ACE2 (10108-H08H), SARS-CoV-2 spike S1+S2 ECD (D614G)-His recombinant protein (40589-V08B4), SARS-CoV spike RBD-His recombinant protein (40150V08B2), MERS-CoV spike RBD-His recombinant protein (40071-V08B1), human coronavirus HKU1 spike-His recombinant protein (40602-V08H), HIV-1 gp120-His recombinant protein (40402-V08H), SARS-CoV-2 spike neutralizing antibody (40592-MM57) and biotin-goat anti-mouse IgG-Fc secondary antibody (SSA013) were obtained from Sino Biological Inc. (China).

\subsection{Cells and pseudoviruses.}

ACE2-transfected $293 \mathrm{~T}$ cells, SARS-CoV-2 S pseudotype HIV, SARS-CoV-2 S with N501Y mutation pseudotype HIV and SARS-CoV-2 S with D614G mutation pseudotype HIV were obtained from Fubio Biotechnology Co., Ltd. (Suzhou, China). DNA sequences used in this work were synthesized by Sangon Biotech (Shanghai, China). The binding buffer (PBS, pH=7.4, including $136.8 \mathrm{mM} \mathrm{NaCl}, 10.1 \mathrm{mM} \mathrm{Na} 2 \mathrm{HPO}_{4}, 2.7 \mathrm{mM} \mathrm{KCl}$, $1.8 \mathrm{mM} \mathrm{KH} 2 \mathrm{PO}_{4}$, and $0.55 \mathrm{mM} \mathrm{MgCl}$ ) was used for aptamer binding or inhibition characterization. Dulbecco's Modified Eagle's Medium (DMEM) was purchased from Hyclone (USA). Fetal bovine serum (FBS) was obtained from BioInd (Israel) and penicillin-streptomycin was from Thermo (USA).

\subsection{Construction of AuNPs functionalized with aptamer cocktail (named as SNAPs).}

Aptamers (CoV2-1C, CoV2-4C and $\mathrm{CoV} 2-6 \mathrm{C} 3)$ used in this work were functionalized into three regions successively ( $\left.5^{\prime}-3^{\prime}\right)$ : thiol-modified at the 5' terminus for bonding to the AuNP surfaces, an 11-nt poly $\mathrm{T}$ for hybridizing 11-nt poly A to form stable sequence ends, and an aptamer sequence used for binding target RBD. For fluorescence quantification and binding characterization, FAM was also included at the 3' terminus. Equal volumes of functionalized $100 \mu \mathrm{M}$ CoV2-1C, CoV2-4C and CoV2-6C3 were mixed to form the aptamer cocktail, which was hybridized with 11-nt poly A at a molar ratio of $1: 1$ at $95{ }^{\circ} \mathrm{C}$ for 10 minutes. Then the product was mixed with AuNPs $(\sim 2.5 \mathrm{nM}, 400 \mu \mathrm{L})$ and placed in a freezer $\left(-20^{\circ} \mathrm{C}\right)$ for $2 \mathrm{~h}{ }^{1}$. After recovering to room temperature, the aptamer-functionalized AuNPs were centrifuged at $10000 \mathrm{rpm}$ for $20 \mathrm{~min}$ with $100 \mathrm{kDa}$ filters to remove excess aptamers. Finally, the aptamer-functionalized AuNPs were dissolved and preserved in PBST (PBS with 0.1\% Tween) for use.

\subsection{Characterization of AuNPs and SNAPs.}

\section{UV-Vis characterization of AuNPs}

The concentration of AuNPs was quantified by UV-visible spectroscopy. The peak SPR wavelength of 5-nm AuNPs was $518 \mathrm{~nm}$. The concentration of AuNPs was calculated by dividing the UV absorbance by the particle extinction coefficient of $5 \mathrm{~nm}$ AuNPs $\left(1.10 \times 10^{7} \mathrm{M}^{-1} \mathrm{~cm}^{-1}\right)$ and then diluted to $2.5 \mathrm{nM}$ for aptamer conjugation.

Transmission electron microscopy (TEM) imaging of AuNPs and SNAPs 
A $10 \mu \mathrm{L}$ aliquot of $2.5 \mathrm{nM}$ AuNPs or aptamer-functionalized AuNPs ( 2.5 nM) was deposited onto a 400-mesh carbon-supported copper grid and allowed to dry. All TEM images were acquired using a JEM1400 electron microscope equipped with a CCD camera. Images of the NPs were analyzed using the instrument's software, and their diameters were calculated using a homemade script compatible with this software.

\section{Dynamic light scattering analysis of AuNPs and SNAPs}

AuNPs and aptamer-functionalized AuNPs were analyzed using a Malvern Panalytical DLS instrument. The sample was illuminated using a $120-\mathrm{mW} \mathrm{He}-\mathrm{Ne}$ laser at $630 \mathrm{~nm}$ at a controlled temperature of $25^{\circ} \mathrm{C}$ and balanced for at least $120 \mathrm{~s}$. The scattered light was measured at an angle of $173^{\circ}$. For the analysis, AuNPs and aptamerfunctionalized AuNPs were prepared at the same concentration as that used in TEM imaging to a final volume of $1.5 \mathrm{~mL}$.

Determination of aptamer loading on 5-nm AuNPs

To quantify the number of aptamer strands attached to AuNPs, FAM-labeled thiol-modified aptamers were used to label AuNPs. The fluorescence intensities of different concentrations of free FAM-labeled aptamer in DTT-PB buffer (0.5 M DTT in $0.18 \mathrm{M}$ sodium phosphate buffer, $\mathrm{pH} 7.85$ ) were measured with a fluorescence spectrometer to establish a standard working curve (Figure S1 A-D). The aptamer-functionalized AuNPs were dissolved in DTTPB buffer to release thiolated DNA. The mixture was then placed in an ultrasonic bath for 10 min and subsequently centrifuged at $10000 \mathrm{rpm}$ for $20 \mathrm{~min}$ with $100 \mathrm{kDa}$ filters to obtain the supernatant. Then the supernatant was measured with a fluorescence spectrometer (Figure S1 E-H), and the concentration of aptamers loading was obtained by comparing its fluorescence intensity against the calibration curve. To ensure the accuracy of the number of DNA loaded on AuNPs, the absorbance at $260 \mathrm{~nm}$ of the release of thiolated DNA was also detected following the Liu's work $^{1}$. Then, with the known concentration of AuNPs, the number of aptamers loaded on each AuNP could be calculated.

\subsection{Cross-competition experiments.}

CoV2-1C, CoV2-4C and CoV2-6C3 were confirmed to bind to different epitopes of SARS-CoV-2 RBD by flow cytometry. FAM labelled $200 \mathrm{nM} \mathrm{CoV} 2-1 \mathrm{C}$ aptamer was used as the target aptamer, while $500 \mathrm{nM}$ non-fluorescent $\mathrm{CoV} 2-4 \mathrm{C}$ or CoV2-6C3 aptamer was added for competition. Only two kinds of aptamers were involved in each competitive experiment. Similarly, FAM labelled CoV2-4C was used to compete with non-fluorescent CoV2-1C aptamer, or with non-fluorescent $\mathrm{CoV} 2-6 \mathrm{C} 3$ aptamer. A similar competitive experiment was conducted with FAM labelled CoV2-6C3 aptamer. The percentage of cross-competition was calculated as Eq. 1: competition efficiency =

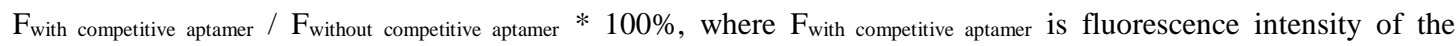
fluorescent aptamer with non-labeled competitor aptamers - fluorescence intensity of background, and $F_{\text {without }}$ competitive aptamer is fluorescence intensity of the same fluorescent aptamer - fluorescence intensity of background.

\subsection{Molecular docking and dynamic simulations.}

The structure of SARS-CoV-2 S protein with a single RBD was obtained from the RCSB PDB data. The processes of aptamer structure prediction, the molecular docking and dynamic simulations were according to our previous work $^{2,3}$. Briefly, the 3D structure with the minimum aptamer free energy was predicted by mfold (http://mfold.rna.albany.edu/?=mfold), and obtained by substituting bases $\mathrm{T}$ for $\mathrm{U}$. And then a refinement process was carried out based on molecular dynamics simulation to relax the aptamer system. Molecular docking was performed with Rosetta after obtaining the 3D structures of aptamers and SARS-CoV-2 S protein from the RCSB PDB data bank (http://www.rcsb.org, ID: 6VSB). The Amber FF99SB and AMBER PARM99 force fields were used for the SARS-CoV-2 S protein and aptamer system, respectively. The final average structure was obtained based on 5000 snapshots, which were extracted from the last $10 \mathrm{~ns}$ trajectory of dynamic simulations using the Gromacs 5.1 
software.

\subsection{Binding affinity and specificity of SNAP.}

To assess the binding affinity of SNAP to wild-typed RBD, mutant RBD with N501Y, mutant spike protein with D614G and mutant RBD with K417N : E484K : N501Y, $0.35 \mathrm{fM}$ corresponding protein-beads were incubated with different concentration of SNAP (0-65 pM) in $100 \mu \mathrm{L}$ binding buffer at $25^{\circ} \mathrm{C}$ for $30 \mathrm{~min}$. The beads were washed twice using binding buffer and suspended in $100 \mu \mathrm{L}$ binding buffer, then the fluorescence intensity of beads measured by flow cytometry (FACS Verse, BD) was recorded as $\mathrm{Y}$ and fitted to the equation $\mathrm{Y}=\mathrm{B}_{\max } * \mathrm{X} /\left(\mathrm{K}_{\mathrm{d}}+\mathrm{X}\right)$ through Origin 8.0 software.

To evaluate the binding specificity of SNAP, SARS-RBD-Ni-beads, MERS-RBD-Ni-beads, HCoV-HKU1Spike-Ni-beads and HIV-1 gp120-Ni beads were incubated with $200 \mathrm{nM}$ random sequence (RS) or $0.5 \mathrm{nM}$ FAMlabeled SNAP in $100 \mu \mathrm{L}$ binding buffer at $25^{\circ} \mathrm{C}$ for $30 \mathrm{~min}$. Then, the beads were washed twice with binding buffer and suspended in $100 \mu \mathrm{L}$ binding buffer, and the fluorescence intensity from beads was measured by flow cytometry (FACSVerse, BD).

To evaluate the binding specificity of SNAP with SARS-CoV-2 pseudoviruses, streptavidin-coated microbeads were washed three times with PBS, and blocked with $5 \%$ out-fat milk powder at $4{ }^{\circ} \mathrm{C}$ overnight. The beads were washed twice and resuspended in $100 \mu \mathrm{L}$ binding buffer (containing 2\% BSA) with $600 \mathrm{nM}$ biotinylated-CoV2-6C 3 at room temperature for $30 \mathrm{~min}$. After washing twice, equivalent amounts pseudoviruses of SARS-CoV-2, HCoVHKU1 or H3N2 were added and incubated for another 1 hour. The beads were washed twice and resuspended in 100 $\mu \mathrm{L}$ binding buffer (containing 2\% BSA) with $1 \mathrm{nM}$ SNAP or $500 \mathrm{nM}$ random sequence at room temperature for 30 min. Finally, the beads were washed twice with PBS (containing 0.1\% BSA) and suspended in $200 \mu \mathrm{L}$ PBS, and the fluorescence intensity from beads was measured by flow cytometry (FACSVerse, BD).

To measure the binding specificity of SNAP against ACE2-293T, HEK-293T and white blood cells, cells were incubated with $200 \mathrm{nM}$ random sequence (RS), $200 \mathrm{nM}$ aptamer cocktail or $0.5 \mathrm{nM}$ FAM-labeled SNAP in $100 \mu \mathrm{L}$ binding buffer at $25^{\circ} \mathrm{C}$ for $30 \mathrm{~min}$. Then, the cells were washed twice and resuspended in $100 \mu \mathrm{L}$ binding buffer for flow cytometry analysis (FACSVerse, BD).

\subsection{RBD neutralization.}

In the RBD protein neutralization assay, $200 \mathrm{nM} / 600 \mathrm{nM}$ CoV2-1C, CoV2-4C, CoV2-6C3, or random sequence, was incubated with $10 \mathrm{ng}$ mouse Fc-tagged SARS-CoV-2 RBD at $25^{\circ} \mathrm{C}$ for $30 \mathrm{~min}$. Then, the mixture was added to ACE2-Ni-beads and incubated for another 1 hour. The beads were washed twice and resuspended in $200 \mu \mathrm{L}$ binding buffer with biotin-goat anti-mouse IgG-Fc secondary antibody. After incubation for another $30 \mathrm{~min}$, the beads were washed twice and resuspended in $200 \mu \mathrm{L}$ binding buffer with SAPE (Streptavidin, R-Phycoerythrin Conjugate from Invitrogen ${ }^{\mathrm{TM}}$ ). To adjust the background signal, the target beads incubated only with SAPE were also included. After the final incubation and washing, the recovered beads were ready for flow cytometry analysis. The percentage of RBD neutralization in the presence of CoV2-1C, CoV2-4C and CoV2-6C3 was compared to RBD binding without aptamers, as calculated by Eq. 2: RBD neutralization $=\left(1-\mathrm{F}_{\text {with aptamer }} / \mathrm{F}_{\text {without aptamer }}\right) * 100 \%$, where $\mathrm{F}_{\text {with aptamer }}$ is fluorescence intensity of ACE2-beads with the aptamer and RBD incubation - fluorescence intensity of ACE2-beads,

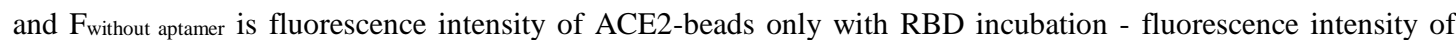
ACE2-beads. The shaded columns represented the concentration of neutralizing reagent at $200 \mathrm{nM}$, unshaded columns represented the concentration of neutralizing reagent $600 \mathrm{nM}$. Assays were repeated as three independent replicates $(n=3)$. 


\subsection{Stability analysis of aptamer cocktail and SNAP in exonuclease solution.}

Aptamer cocktail $(600 \mathrm{nM})$ or SNAPs $(1 \mathrm{nM})$ were incubated with $0,1,2.5,5,10,40$ or 80 U exonuclease I (Exo I) for $30 \mathrm{~min}$ at $37^{\circ} \mathrm{C}$. Then, samples were heated at $80^{\circ} \mathrm{C}$ for $20 \mathrm{~min}$ to denature the enzyme. After cooling to room temperature, $10 \mu \mathrm{L}$ samples were loaded onto $8 \%$ polyacrylamide gel in $1 \times \mathrm{TBE}$ buffer and run at $80 \mathrm{~V}$ for $40 \mathrm{~min}$. After electrophoresis, the gels were stained with nucleic acid staining (Gel Red) and analyzed with a molecular imager (BIO-RAD).

\subsection{Pseudovirus neutralization analysis.}

In Figure 3A, 4A, 4C and 4E, to determine the neutralizing ability of antibody, free aptamers CoV-1C, CoV-6C3, aptamer cocktail and their functionalized AuNPs (CoV-1C-AuNPs, 6C3-AuNPs, AuNPs cocktail), the pseudovirus neutralization assay was performed as previously described ${ }^{4,5}$. First, DMEM supplemented with $10 \%$ fetal bovine serum and $5 \mu \mathrm{L}$ pseudoviruses per well were preincubated with $340 \mathrm{nM}$ aptamer or $1 \mathrm{nM}$ aptamer-functionalizednanoparticles at $37^{\circ} \mathrm{C}$. One hour later, the mixtures were added to ACE2-expressing 293T cells. After six hours of infection, the culture medium was replaced with fresh medium and then incubated at $37^{\circ} \mathrm{C}$ for another $48 \mathrm{~h}$. To exclude an unspecific effect due to the high local concentration of aptamers, SNAP equipped with 54-nt random sequence or 1:1:1 mixture of scrambled $\mathrm{CoV}-1 \mathrm{C}, \mathrm{CoV}-4 \mathrm{C}$ and $\mathrm{CoV}-6 \mathrm{C} 3$ were introduced and conducted as above. GFP expression in infected ACE2-transfected $293 \mathrm{~T}$ cells, which were seeded at $1.6 \times 10^{4}$ cells/well in a $384-$ well tissue-culture plate, was determined by fluorescence microscopy. Possible reasons for the need for a high number of seeded cells include: 1) the adhesion ability of selected ACE2 expressed HEK-293T cells is weaker than that of tumor cells; 2) cell proliferation will be affected by virus infection; 3) the multi-step of fluid transfer in the experiment may cause some cell loss.

In Figure 3B, 4B, 4D and 4F, the luciferase activity of ACE2-transfected 293T cells, which were plated at 1.0×104 cells/well in a 96-well tissue culture plate, was measured on a Multimode Plate Reader (SpectraMax® iDS). The resulting curves were analyzed by nonlinear regression. The $\mathrm{IC}_{50}$ values were obtained using Prism software, version 8.3.0 (GraphPad), and obtained by fitting data to an [inhibitor] vs response -variable slope. The detailed information of fitting equation was provided by GraphPad (https://www.graphpad.com/guides/prism/latest/curvefitting/reg_dr_inhibit_variable.htm). These experiments were performed three times.

\subsection{Transmission electron microscopy (TEM) and cryo-electron microscopy (cryo-EM) imaging of the binding mode between SNAPs and the pseudovirus.}

For TEM imaging, $2.5 \mathrm{nM}$ SNAPs were incubated with $2.18 \times 10^{11}$ particles $/ \mathrm{mL}$ pseudovirus at $37{ }^{\circ} \mathrm{C}$ for $1 \mathrm{~h}$. Five $\mu \mathrm{L}$ of the mixture was deposited onto glow-discharged 300-mesh carbon-supported copper grids (Agar scientific) for $1 \mathrm{~min}$ at room temperature. Then, the grids were blotted with filter paper, washed once with MilliQ water and immersed into a $2 \% \mathrm{w} / \mathrm{v}$ uranyl acetate solution with $25 \mathrm{mM} \mathrm{NaOH}$ for $1 \mathrm{~min}$. The blotted grids were airdried and imaged using a transmission electron microscope (H-7000 FA, Hitachi) at an acceleration voltage of 100 $\mathrm{kV}$ and equipped with a CCD camera.

For cryo-EM microscopy, $12 \mathrm{nM}$ SNAPs were incubated with $2.18 \times 10^{11}$ particles $/ \mathrm{mL}$ pseudovirus at $37^{\circ} \mathrm{C}$ for $1 \mathrm{~h}$. Four $\mu \mathrm{L}$ of the mixture was deposited onto Quantifoil copper grids (Agar Scientific), blotted, frozen in liquid ethane with a Vitrobot Mark IV (ThermoFisher Scientific, USA) and transferred to liquid nitrogen. The grid was then quickly placed in a cryogenic stage and kept at $-180{ }^{\circ} \mathrm{C}$. Micrographs were collected using cryo-EM (Talos F200C G2, Thermo Fisher Scientific, USA) operating at $200 \mathrm{kV}$ with a CCD camera. It may be because the cryoEM microscopy sample preparation is made with $\mathrm{Mg} 2^{+}$-free PBS buffer, and the operation such as washing off the supernatant with filter paper and freezing in liquid ethane is required, which results a small amount of SNAP binding to the virus. 


\subsection{Cell viability analysis.}

ACE2-transfected $293 \mathrm{~T}$ cells $\left(1 \times 10^{4}\right)$ were cultured in a 96-well plate overnight. After $12 \mathrm{~h}$, different concentrations of SNAPs were added to the monolayer of cells. After $48 \mathrm{~h}$ of addition, cell viability was measured using the CCK-8 kit (Beyotime, China). The absorbance was read at $450 \mathrm{~nm}$ by a microplate reader (SpectraMax iDS). Six independent experiments were conducted for cell viability determination.

\subsection{Authentic viral neutralization assays.}

All experiments involving live SARS-CoV-2 (GenBank: MT835143.1,
https://www.ncbi.nlm.nih.gov/nuccore/MT835143) followed the approved standard operating procedures of the University of Hong Kong Biosafety Level-3 facility. The mixtures of serially diluted aptamers and real viruses were incubated at $37^{\circ} \mathrm{C}$. Following incubation for $1 \mathrm{~h}$, the mixtures were added to Vero E6 cells. After another hour of incubation, the supernatant was removed and replaced with DMEM medium with 5\% FBS. After $48 \mathrm{~h}$ incubation at $37^{\circ} \mathrm{C}$, cells were homogenized in $100 \mu \mathrm{L}$ RNAzol solution for the next analysis. The viral mRNA (S gene) and host gene (GAPDH) were detected in a Light Cycle 480 instrument (Roche), and gene expression was measured by quantitative PCR (RT-qPCR) using the SYBR green premix Kit (Takara). The relative copy number of viral mRNA compared to that of host gene mRNA was analyzed by nonlinear regression to calculate the IC 50 value using Prism software, version 8.3.0 (GraphPad). For immunofluorescence analysis, Vero E6 cells were fixed and incubated in $70 \%$ ethanol at $4{ }^{\circ} \mathrm{C}$ overnight for permeabilization. The expression of nucleocapsid protein from SARS-CoV-2 virus was detected by using $\mathrm{N}$ protein primary antibody (Cat: 40143-MM05TA, SinoBiological) for $1 \mathrm{~h}$ at $37^{\circ} \mathrm{C}$. After washing with PBST, slides were incubated with secondary antibody (anti-mouse, Alexa Fluor ${ }^{\circledR} 594$ ) for 1 hour at room temperature. Then slides were mounted with mounting buffer containing Hoechst. Images were acquired with the LSM780 confocal microscope and analyzed with ZEN software.

\subsection{Imaging of SNAP and cell binding.}

ACE2-expressing $293 \mathrm{~T}$ cells were seeded in a glass bottom dish for cell culture at least $24 \mathrm{~h}$. Then, $2.5 \mathrm{nM}$ SNAP labeled with FAM-labeled thiol-modified aptamers (CoV2-1C, CoV2-4C and CoV2-6C3) was added and incubated with ACE2-expressing 293T cells for 4 hours. After $4 \mathrm{~h}$, the supernatant liquid was discarded to remove the unbound SNAP, and cells were washed three times with PBS. Before imaging assay with a confocal microscopy (Leica TCS SP8), the cells were stained with Hoechst 33342 for 15 min.

\subsection{Proinflammatory cytokines analysis in vivo.}

All animal procedures were performed in accordance with the National Institute of Health Guidelines for the care and use of laboratory animals and were approved by the Institutional Animal Care and Use Committee of Xiamen University (XMULAC20190162). BALB/c mice were obtained from the Laboratory Animal Center of Xiamen University. BALB/c mice were randomized into three groups $(n=3$, each group) and treated by tail-vein injection with SNAP or control samples. In group 1 , SNAP ( $8 \mathrm{nM}$ in $150 \mu \mathrm{L}$ saline) was tail-vein injected, followed by standard orbital blood collection and sacrifice by anesthesia, after which cervical dislocation was performed at 24 hours post-injection. In groups 2 and 3, SNAP ( $8 \mathrm{nM}$ in $150 \mu \mathrm{L}$ saline) or $150 \mu \mathrm{L}$ saline was tail-vein injected, followed by standard orbital blood collection and sacrifice by anesthesia, after which cervical dislocation was performed at 7 days post-injection. The blood was placed overnight in a $1.5 \mathrm{~mL}$ EP tube, centrifuged at $3000 \mathrm{RPM}$ for $10 \mathrm{~min}$ to remove cells, followed by collection of $100 \mu \mathrm{L}$ serum for blood biochemistry analysis. All blood biochemistry analyses were performed by Wayenbio Company, Shanghai, China. 


\section{Supporting Figures}
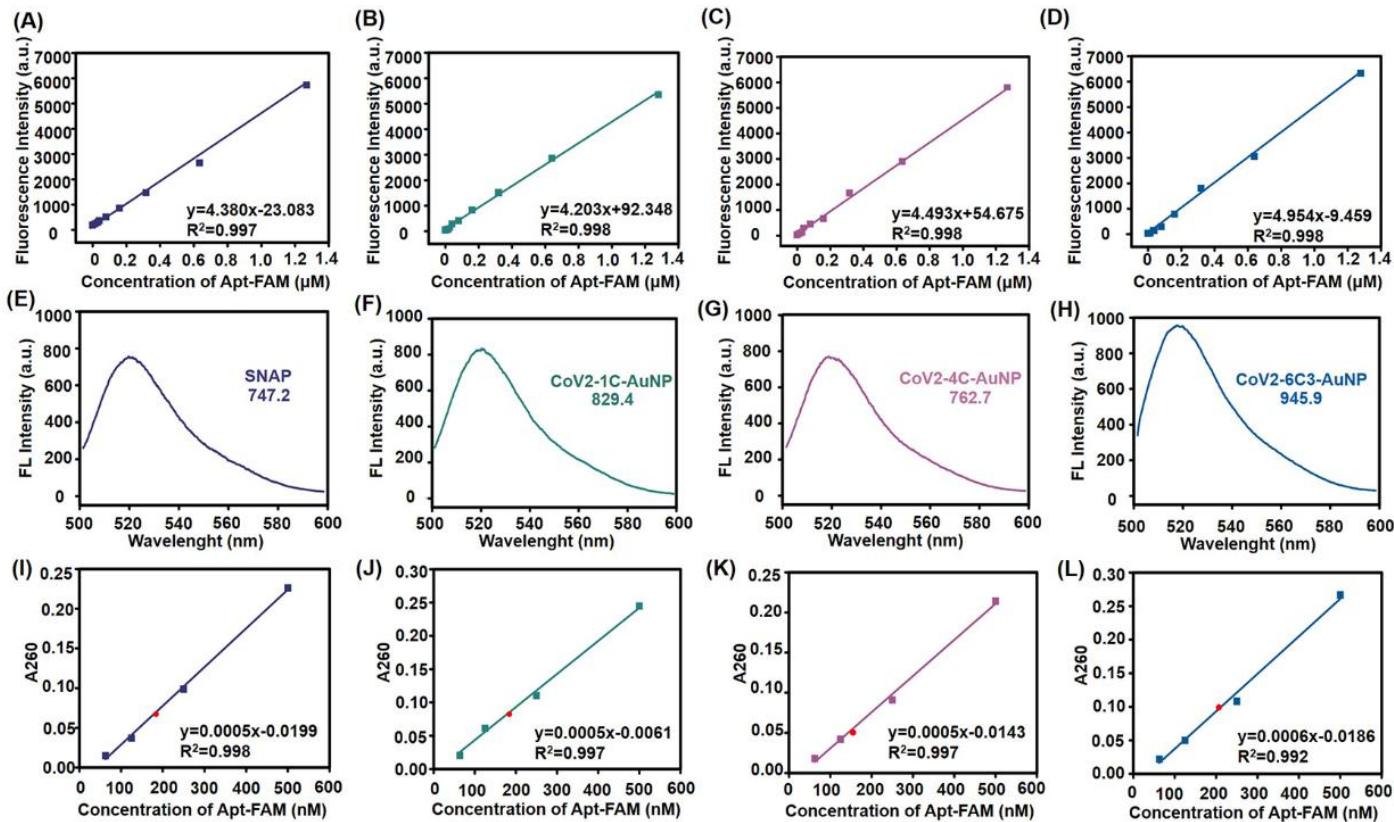

Figure S1. Quantification of aptamer loading density on AuNP. (A-D) Standard curve of free FAM-labeled aptamer in DTT-PB buffer measuring the fluorescence at $520 \mathrm{~nm}$. (E-H) Fluorescence spectra of FAM-labeled Apt-AuNP in DTT-PB buffer. (I-L) Standard curve of free FAM-labeled aptamer measuring the UV-vis absorbance at $260 \mathrm{~nm}$, and the absorbance of Aptamer-FAM was shown as red dots. Purple line represents SNAP, green line represents CoV2-1C-AuNP, pink line represents CoV2-4C-AuNP, and blue line represents CoV2-6C3-AuNP.

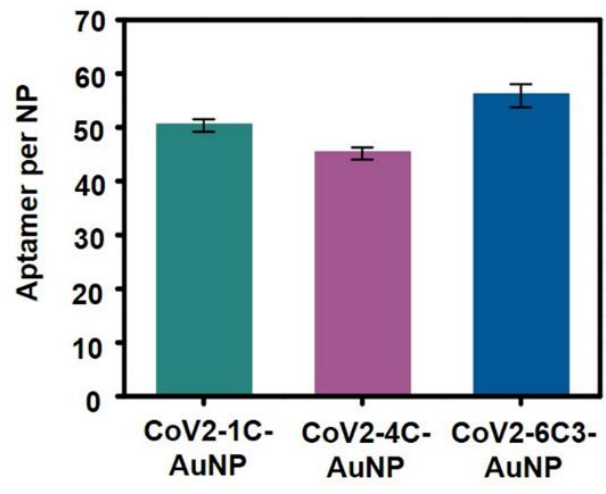

Figure S2. The number of individual aptamers (CoV2-1C, CoV2-4C and CoV2-6C3) on each AuNPs. Assays were repeated three times. 
(A)

RBD-Beads

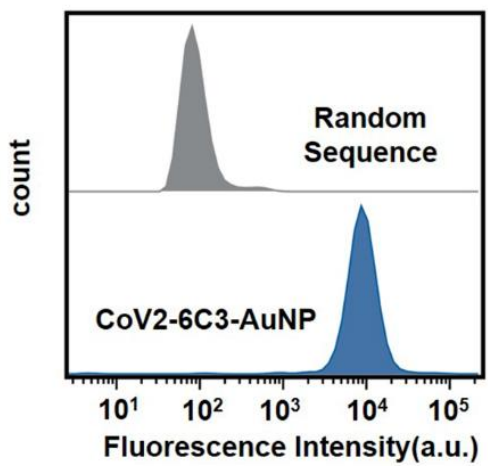

(B)

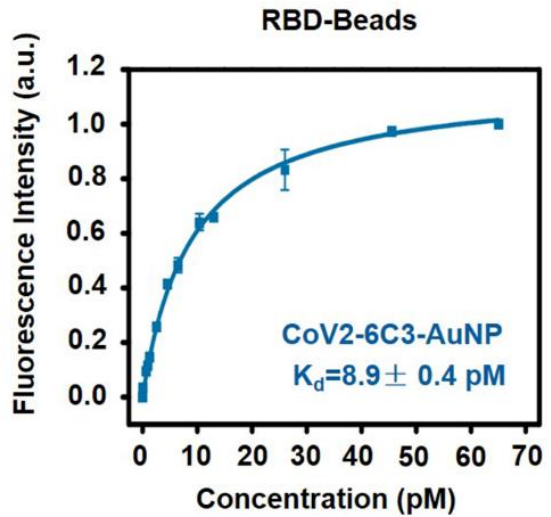

Figure S3. Binding performance of CoV2-6C3-AuNP to SARS-CoV-2 RBD. (A) Flow cytometry to investigate the binding performance of CoV2-6C3-AuNP. (B) Binding curve of CoV2-6C3-AuNP against SARS-CoV-2 RBD-bead. The SNAP concentration was the corresponding concentration of AuNPs. Assays were repeated three times.
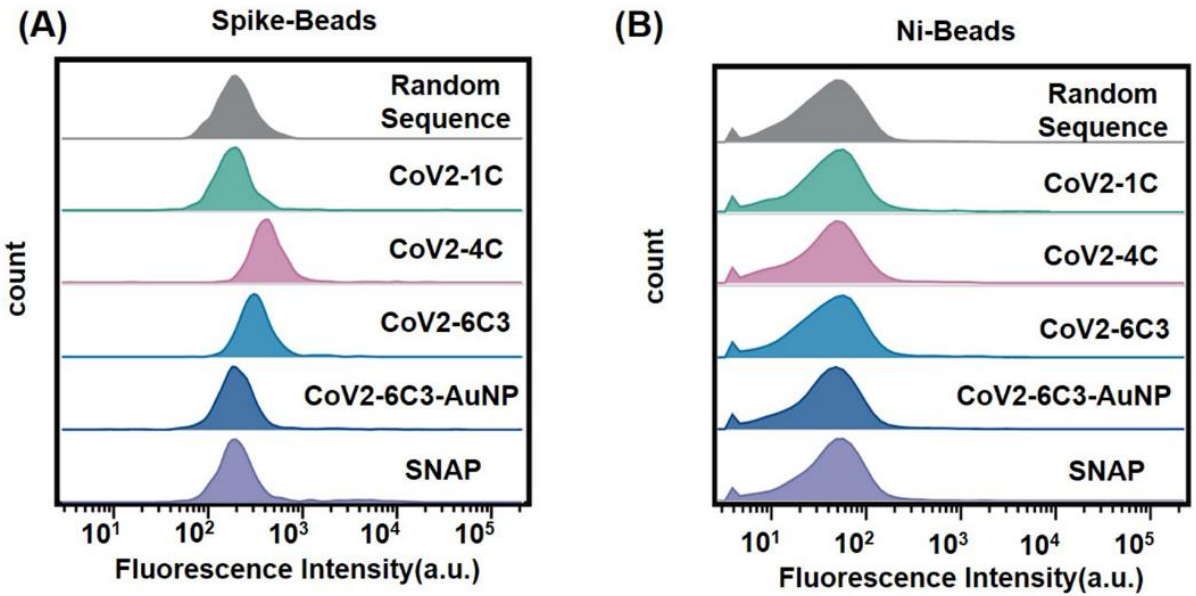

Figure S4. Binding performance of free aptamers and their functionalized AuNPs to Spike-beads and Ni-beads. Flow cytometry to monitor the binding performance with (A) Spike-beads (target beads) and (B) Ni-beads (control beads).

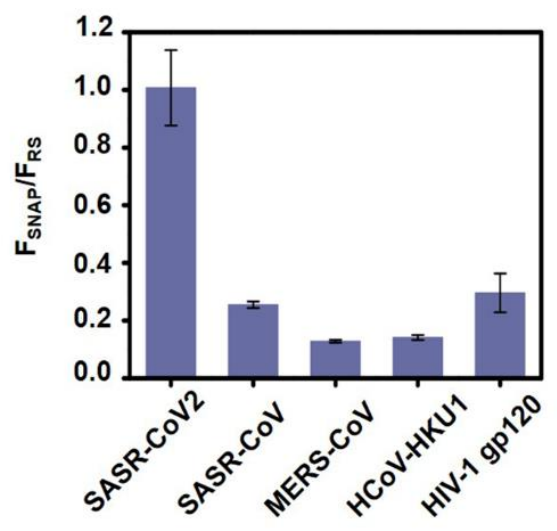

Figure S5. Selectivity study of SNAP against SARS-CoV-2-RBD, SARS-CoV-RBD, MERS-CoV-RBD, HCoVHKU1-Spike, and HIV-1 gp120, calculating as the equation: fluorescence ratio = SNAP (fluorescence intensity) $/$ random 
sequence (fluorescence intensity). The fluorescence ratio of SARS-CoV-2 set as 1, then assess the occupation rate of other control targets compared to SARS-CoV-2.

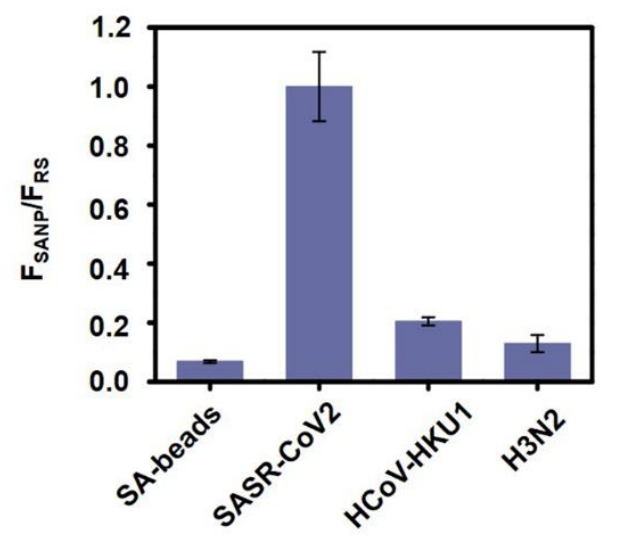

Figure S6. Selectivity study of SNAP against pseudoviruses of SARS-CoV-2, HCoV-HKU1 or H3N2. The sandwich assay was conducted with streptavidin-coated microbeads (SA-beads). The biotinylated CoV2-6C3 aptamers immobilized onto SA beads were used to capture viral particles. Then, fluorescent labelled SNAP and random sequence were added to detect the pseudovirus bound on the SA-beads, and then analyzed by flow cytometry. As control, SNAP and random sequence incubated only with SA-beads were included. The binding of SNAP against pseudoviruses of SARS-CoV-2, HCoV-HKU1 or H3N2 was calculated as the equation: fluorescence ratio $=$ SNAP (fluorescence intensity) / random sequence (fluorescence intensity). The fluorescence ratio of SARS-CoV-2 set as 1, then assess the occupation rate of other control targets compared to SARS-CoV-2.

(A)

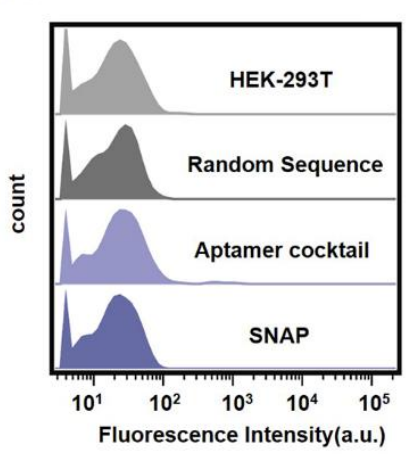

(B)

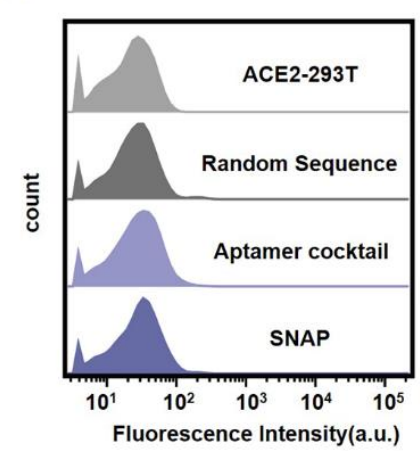

(C)

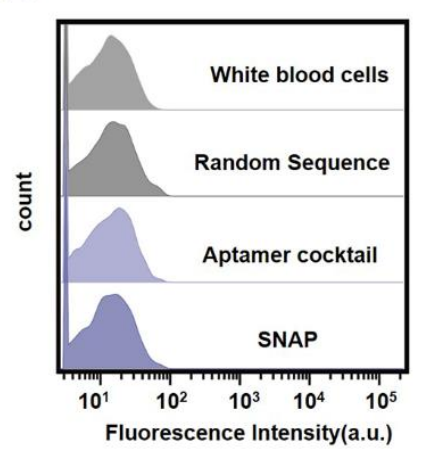

Figure S7. Binding performance of aptamer cocktail and SNAP to HEK-293T, ACE2 expressed HEK-293T cells and white blood cells. 


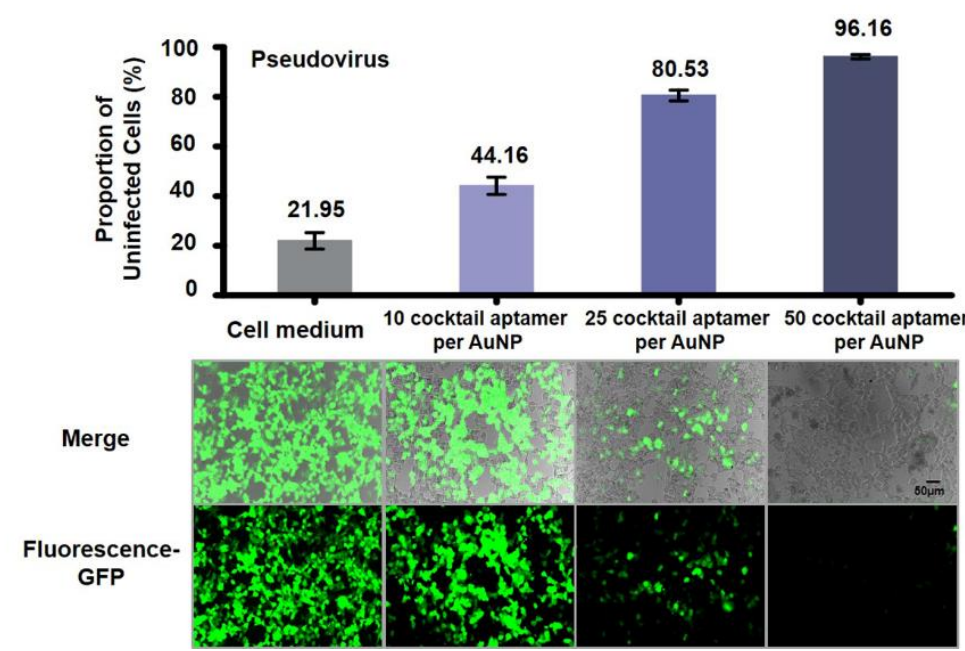

Figure S8. The neutralization of wild-type pseudovirus infection by SNAP with different aptamer densities (10 cocktail aptamers per SNAP, 25 cocktail aptamers per SNAP, and 50 cocktail aptamers per SNAP).

Pseudovirus

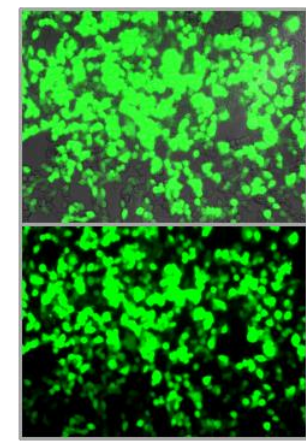

AuNP

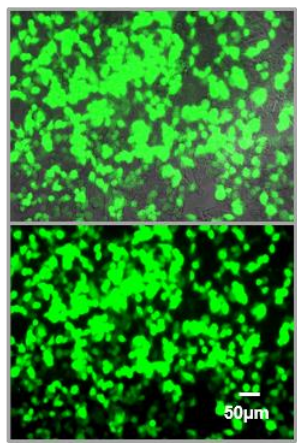

Figure S9. Fluorescence microscopy imaging of the AuNPs effect on neutralizing the pseudovirus infection.

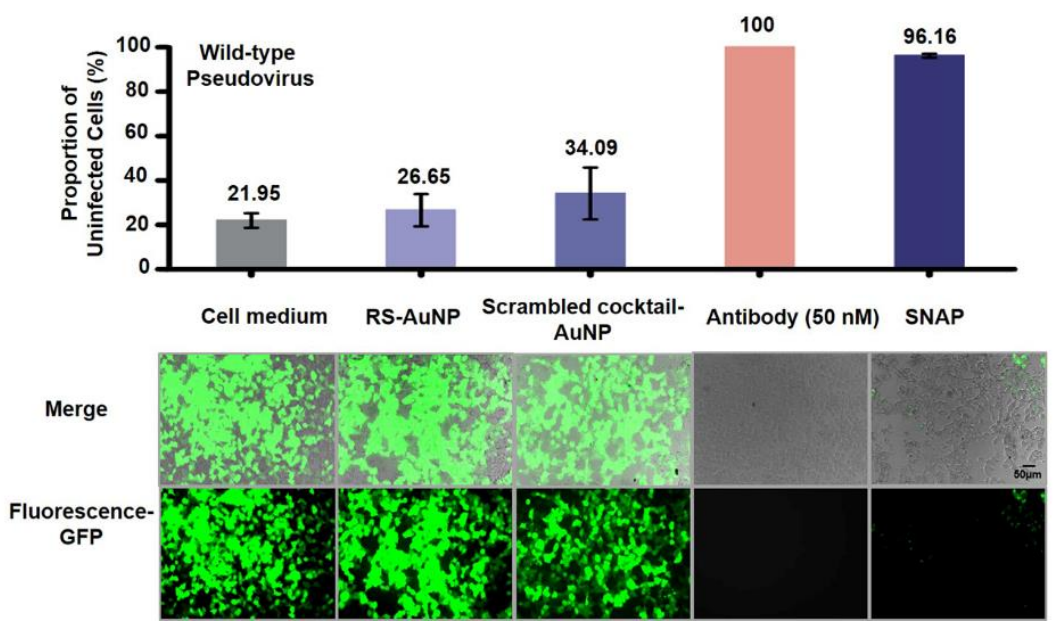

Figure S10. Neutralization efficiency of the $1 \mathrm{nM}$ RS-AuNP, scrambled cocktail-AuNP, SNAP or $50 \mathrm{nM}$ antibody against pseudovirus infection (50 random sequences per AuNP, 50 scrambled cocktail aptamers per AuNP, and 50 cocktail aptamers per SNAP). 


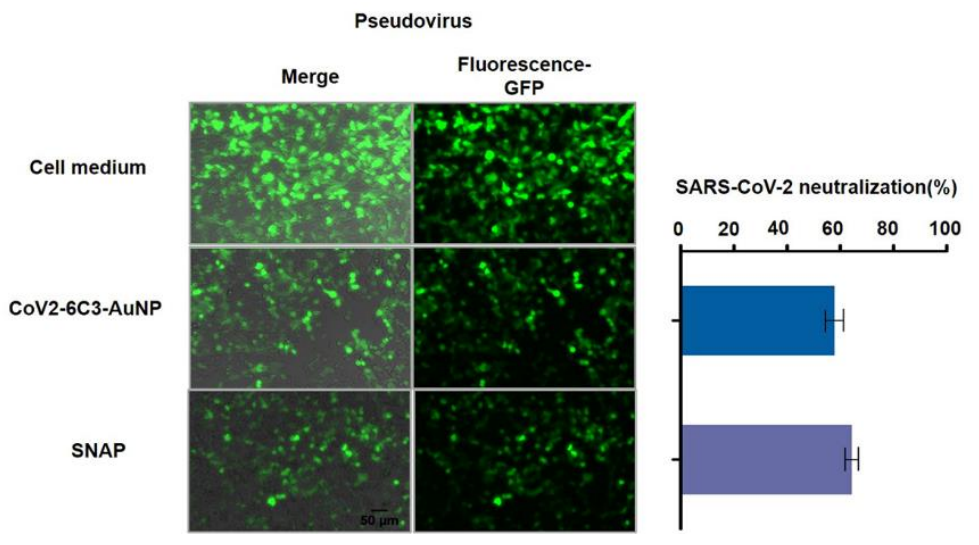

Figure S11. The neutralization of wild-type pseudovirus infection by CoV2-6C3-AuNP and SNAP with the scaffold of 18-nm AuNP.
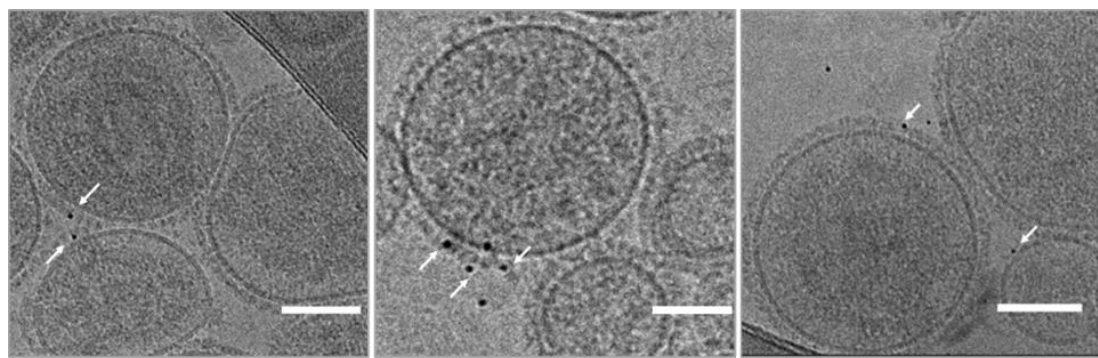

Figure S12. Cryo-electron microscopy micrographs of interaction of SNAP and SARS-CoV-2 pseudovirus. The concentration of virus is about 100 times that of SNAP. The scale bar is $50 \mathrm{~nm}$. It may be because the cryo-EM microscopy sample preparation is made with magnesium-free PBS buffer, and the operation such as washing off the supernatant with filter paper and freezing in liquid ethane is required, which results a small amount of SNAP binding to the virus

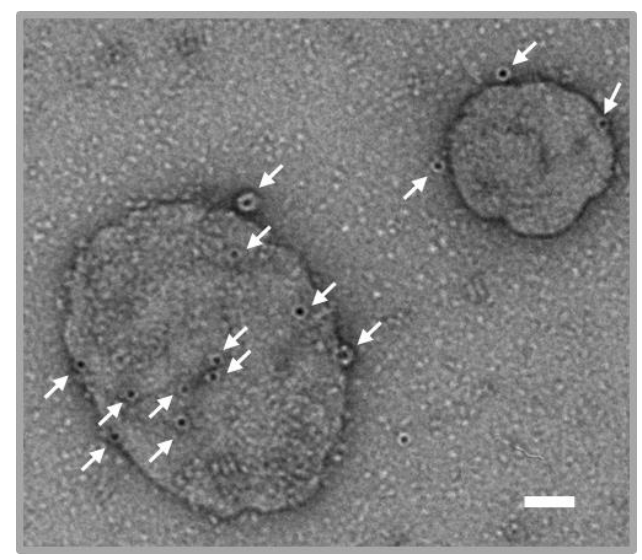

Figure S13. TEM micrographs of SNAP binding to SARS-CoV-2 pseudovirus. The scale bar is $50 \mathrm{~nm}$. 




Figure S14. Confocal imaging of the HEK-293T cell treated without or with SNAP. Hoechst and FAM stain the nucleus and SNAP, respectively.

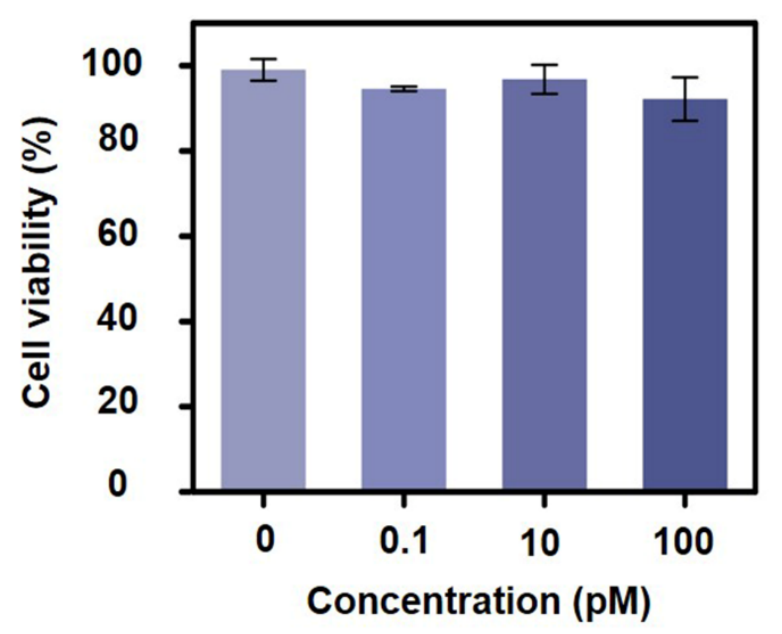

Figure S15. Viability of ACE2-transfected 293T cells after treatment with different concentrations of SNAP. 


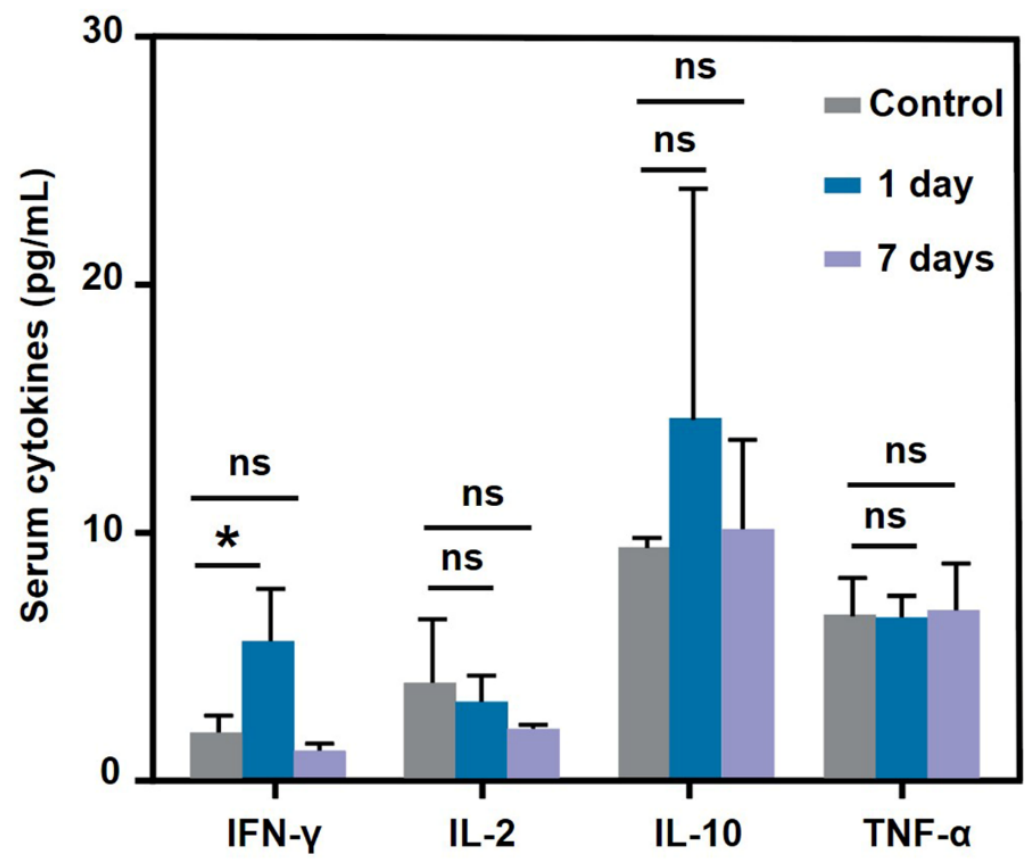

Figure S16. Time-course effects of SNAP on the proinflammatory cytokines in mouse serum, showing no significant difference between the values of SNAP injection and control sample. (-t-test: $* \mathrm{P}<0.05$ versus control group).

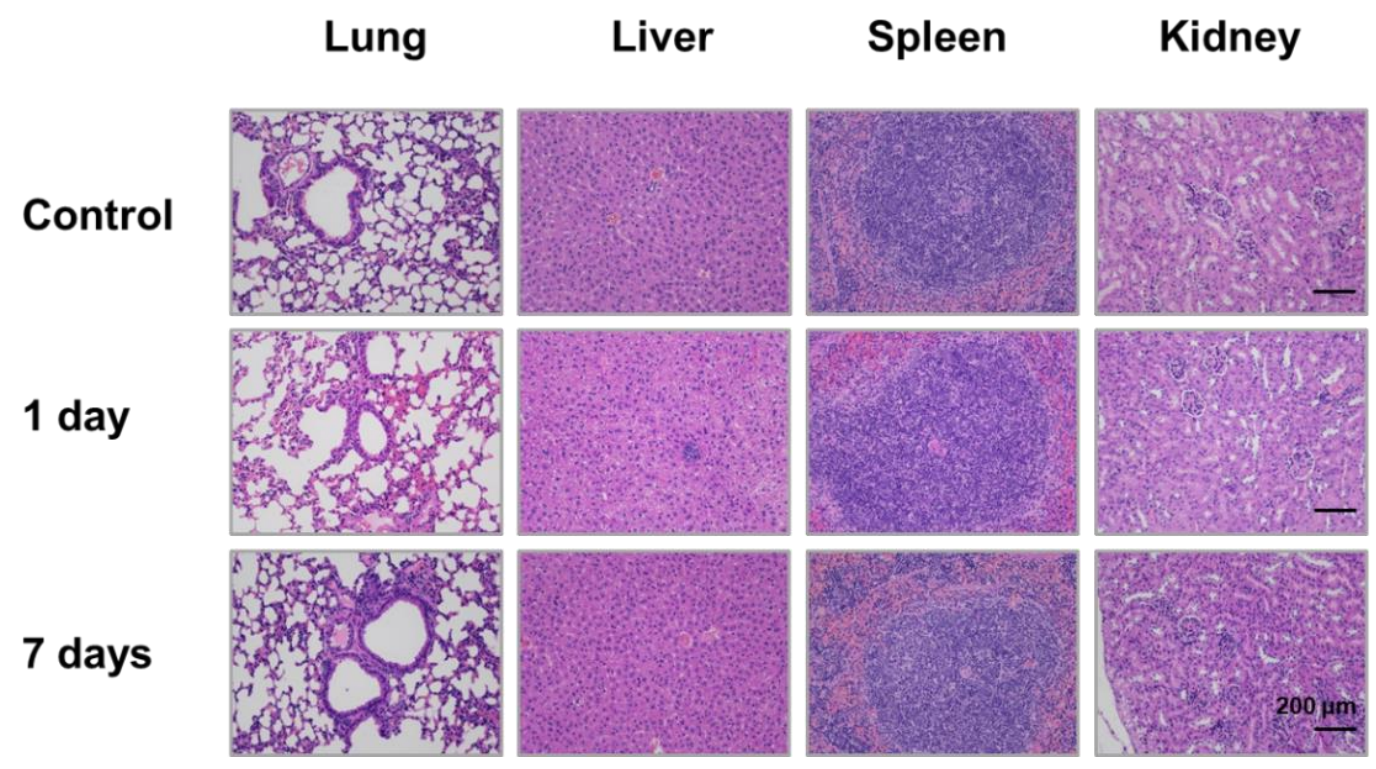

Figure S17. Histology of animal organs following SNAP administration. Animals were intravenously injected with $8 \mathrm{nM}$ SNAP. Organs were harvested 1 day or 7 days post-injection (DPI), fixed using $10 \%$ buffered formalin and embedded in paraffin blocks, and sectioned for histological examination using a hematoxylin/eosin stain. Organs from untreated animals were analyzed as negative control. No acute tissue damage, necrosis, or inflammation was observed in treated animals relative to control. 
(A)

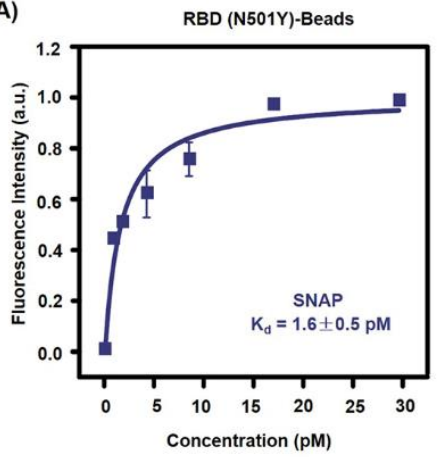

(B)

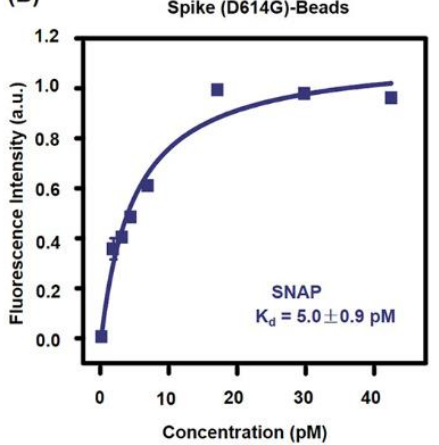

(C)

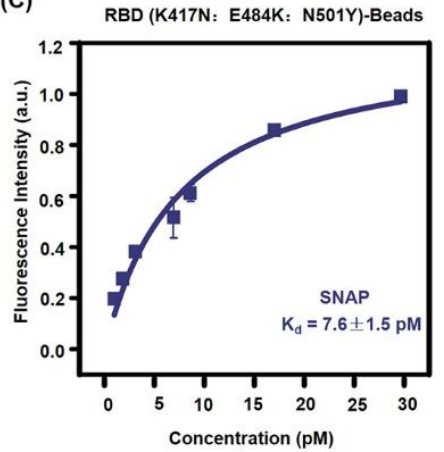

Figure S18. Binding affinity of SNAP against RBD with N501Y mutation-bead (A), spike protein with D614G mutation-bead (B) and RBD with K417N : E484K : N501Y mutation-bead (C). Assays were repeated three times. The relationship between affinity and inhibition ability is not completely consistent, which may be because some of the three aptamers (CoV2-1C and CoV2-4C) have high affinity but no good inhibition ability.

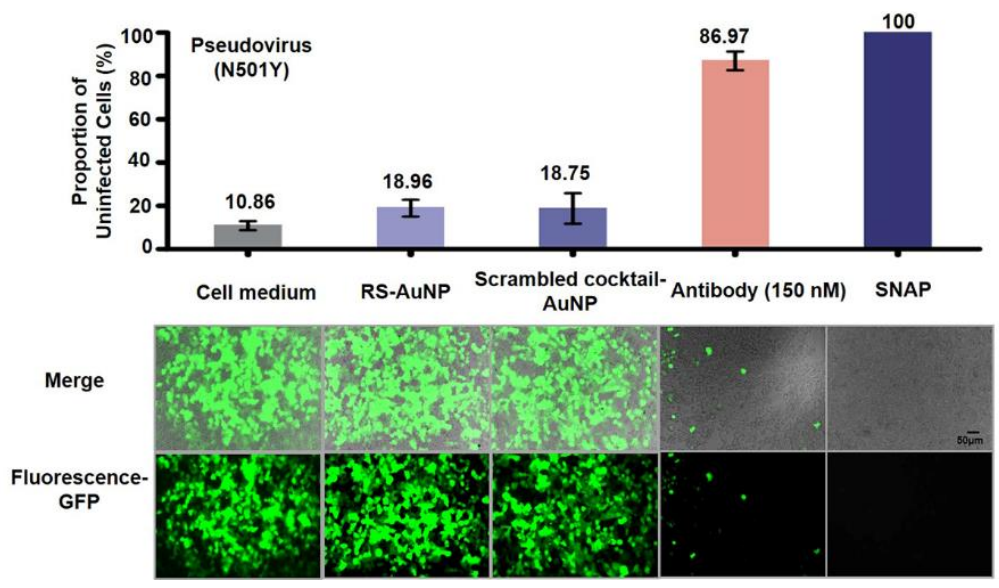

Figure S19. Neutralization efficiency of the 3 nM RS-AuNP, scrambled cocktail-AuNP, SNAP or $150 \mathrm{nM}$ antibody against the mutant pseudovirus (N501Y) infection (50 random sequences per AuNP, 50 scrambled cocktail aptamers per AuNP, and 50 cocktail aptamers per SNAP).

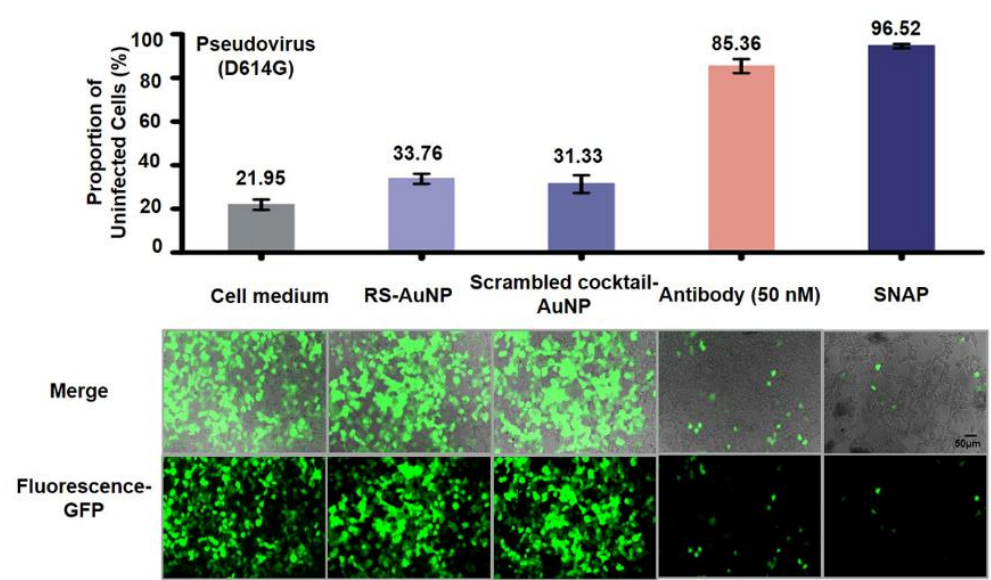

Figure S20. Neutralization efficiency of the $1 \mathrm{nM}$ RS-AuNP, scrambled cocktail-AuNP, SNAP or $50 \mathrm{nM}$ antibody against the mutant pseudovirus (D614G) infection (50 random sequences per AuNP, 50 scrambled cocktail aptamers per AuNP, and 50 cocktail aptamers per SNAP). 


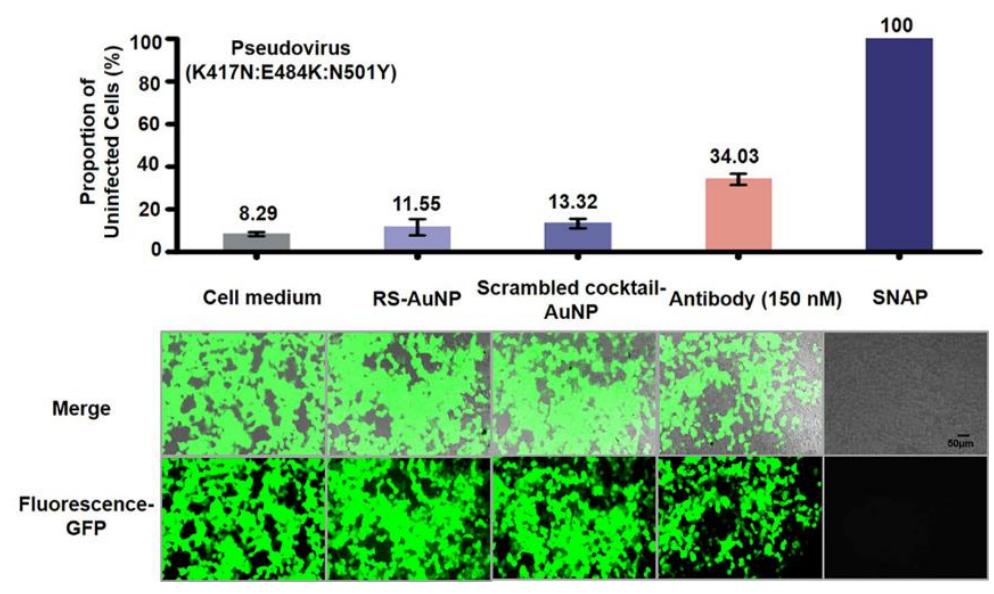

Figure S21. Neutralization efficiency of the 3 nM RS-AuNP, scrambled cocktail-AuNP, SNAP or $150 \mathrm{nM}$ antibody against the mutant pseudovirus (K417N : E484K : N501Y) infection (50 random sequences per AuNP, 50 scrambled cocktail aptamers per AuNP, and 50 cocktail aptamers per SNAP).

Table S1 Sequences used in this work.

\begin{tabular}{ll}
\hline Name & Sequence (5'-3') \\
\hline CoV2-1C & SH C6- \\
& TTTTTTTTTTCAGCACCGACCTTGTGCTTTGGGAGT \\
& GCTGGTCC \\
& AAGGGCGTTAATGGACA-(FAM) \\
CoV2-4C & SH C6- \\
& TTTTTTTTTTATCCAGAGTGACGCAGCATTTCATCGG \\
& GTCCAAAA \\
& GGGGCTGCTCGGGATTGCGGATATGGACACGT(FAM) \\
CoV2-6C3 & SH C6- \\
& TTTTTTTTTTCGCAGCACCCAAGAACAAGGACTGCT \\
& TAGGATTGC \\
& GATAGGTTCGG-(FAM) \\
Poly A-11 & AAAAAAAAAAA \\
\hline
\end{tabular}

Table S2 Binding Sites of RBD-ACE2, RBD-aptamer, and mutation sites in mutant strains used in this work.

\begin{tabular}{|c|c|c|c|c|c|c|c|}
\hline $\begin{array}{l}\text { The binding sites of RBD-ACE2 } \\
\text { (Science, 2020,367, 1444-1448) }\end{array}$ & K417 & Y453 & Q474 & F486 & Q498 & $\mathrm{T} 500$ & N501 \\
\hline $\begin{array}{l}\text { The simulated binding sites of RBD- CoV2- } \\
\text { 1C (Anal. Chem., 2020, 92, 9895-9900) })^{2}\end{array}$ & N370 & A372 & F374 & S375 & N437 & T500 & Q506 \\
\hline $\begin{array}{l}\text { The simulated binding sites of RBD- CoV2- } \\
\text { 4C (Anal. Chem., 2020, 92, 9895-9900) }\end{array}$ & Q409 & K417 & Y421 & & & & \\
\hline $\begin{array}{l}\text { The simulated binding sites of RBD- CoV2- } \\
\text { 6C3 (Angew. Chem. Int. Ed., 2021,133, } \\
\text { 10354-10360) }\end{array}$ & Y449 & $\mathrm{R} 454$ & R457 & Q474 & G485 & F486 & Y489 \\
\hline Mutation sites in mutant strains & $\mathrm{K} 417 \mathrm{~N}$ & E484K & N501Y & D614G & & & \\
\hline
\end{tabular}


Table S3 Variants involved in this work.

\begin{tabular}{cccc}
\hline WHO label & $\begin{array}{c}\text { Pango } \\
\text { lineage }\end{array}$ & $\begin{array}{c}\text { Mutation site on } \\
\text { RBD }\end{array}$ & $\begin{array}{c}\text { Earliest } \\
\text { documented } \\
\text { samples }\end{array}$ \\
\hline Blpha & B.1.1.7 & N5014G* & $\begin{array}{c}\text { Northern Italian, } \\
\text { Jan-2020 } \\
\text { United Kingdom, } \\
\text { Beta }\end{array}$ \\
& B.1.351 & K417N: & $\begin{array}{c}\text { Sep-2020 } \\
\text { South Africa, } \\
\text { Eay-2020 }\end{array}$ \\
\hline
\end{tabular}

* SARS-CoV-2 with mutation D614G occurred in other S1 site except RBD, existing in the non-RBD area of all variants of concern. Information from WHO (https://www.who.int/en/activities/tracking-SARS-CoV-2-variants/).

\section{References}

1. Liu, B.; Liu, J., Freezing Directed Construction of Bio/Nano Interfaces: Reagentless Conjugation, Denser Spherical Nucleic Acids, and Better Nanoflares. J. Am. Chem. Soc. 2017, 139 (28), 9471-9474.

2. Song, Y.; Song, J.; Wei, X.; Huang, M.; Sun, M.; Zhu, L.; Lin, B.; Shen, H.; Zhu, Z.; Yang, C., Discovery of Aptamers Targeting the Receptor-Binding Domain of the SARS-CoV-2 Spike Glycoprotein. Anal. Chem. 2020, 92 (14), 9895-9900.

3. Sun, M.; Liu, S.; Wei,X.; Wan, S.; Huang, M.; Song, T.; Lu, Y.; Weng, X.; Zhu, Z.; Chen, H.; Song, Y.; Yang, C., Aptamer Blocking Strategy Inhibits SARS-CoV-2 Virus Infection. Angew.Chem. Int. Ed. 2021, 60 (18), 1026610272.

4. Pallesen, J.; Wang, N.; Corbett, K.; Wrapp, D.; Kirchdoerfer, R.; Turner, H; Cottrell, C.; Becker, M.; Wang, L.; Shi, W., Immunogenicity and structures of a rationally designed prefusion MERS-CoV spike antigen. Proc. Natl. Acad. Sci. 2017, E7348-E7357

5. Wang, L.; Shi, W.; Joyce, M.; Modjarrad, K.; Zhang, Y.; Leung, K.; Lees, C.; Zhou, T.; Yassine, H.; Kanekiyo, M., Evaluation of candidate vaccine approaches for MERS-CoV. Nat. Commun. 2015, 6, 7712.

6. Yan, R.; Zhang, Y.; Li, Y.; Xia, L.; Guo, Y.; Zhou, Q. J. S., Structural basis for the recognition of SARS-CoV-2 by full-length human ACE2. Science 2020, 367 (6485), 1444-1448.5. 